\title{
Aplicación de un Modelo Evaluativo de Usabilidad a Recursos Educativos Virtuales Sobre Química
}

Recibido: 15 Febrero 2014 - Revisado: 30 Abril 2014 Aceptado: 30 Mayo 2014 - Publicado: 30 Julio 2014

\section{Nora R. Nappa}

Profesor Asociado, Instituto de Investigaciones en Educación de las Ciencias Experimentales (IIECE). Nacional de San Juan, San Juan, Argentina. noranappa@yahoo.com.ar

\section{Nora E. Herrera}

Profesor Titular, Instituto de Investigaciones en Educación de las Ciencias Experimentales (IIECE). Departamento de Física y de Química. Universidad Nacional de San Juan, San Juan, Argentina. nora edith herrera@yahoo.com.ar

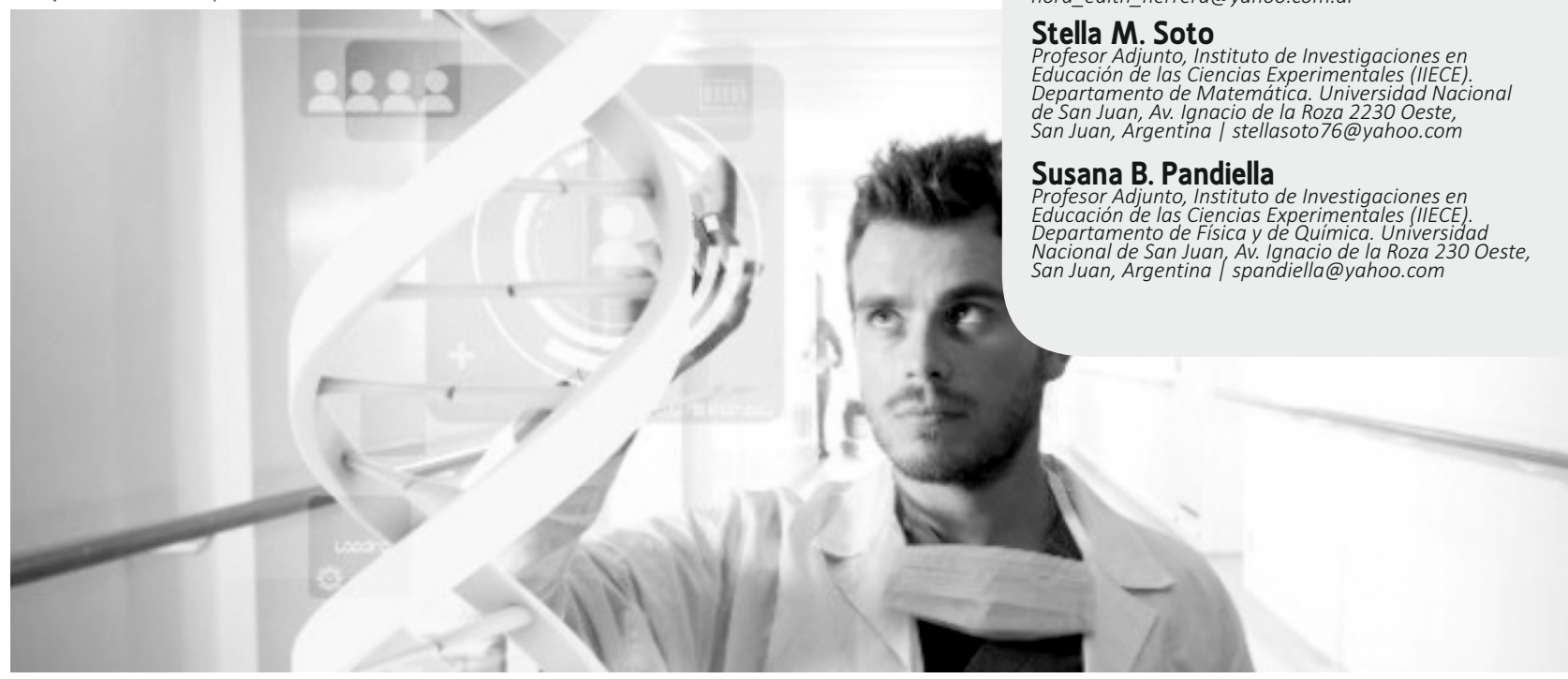

Resumen: La incorporación de las Tecnologías de la Información y Comunicación en la enseñanza permite a los alumnos desarrollar nuevas competencias necesarias para desenvolverse en la Sociedad del Conocimiento. La elección y el uso de recursos de la web necesitan también que los usuarios sean críticos y posean las competencias para evaluar el material que utilizan para el aprendizaje. El propósito de este trabajo fue la incorporación en el aula de recursos educativos abiertos de química y su evaluación de la usabilidad por parte de los estudiantes, empleando un cuestionario propuesto por autores españoles (Marzal et al., 2008). La muestra estuvo formada por dos cursos correspondientes a alumnos de 15 años de edad promedio, de Nivel Secundario de Instituciones Escolares de la Provincia de San Juan, República Argentina. Los resultados obtenidos de la evaluación de la usabilidad muestran que para los alumnos, los recursos resultaron fáciles de usar y de aprender a manejar, con contenidos claramente organizados y con imágenes apropiadas y atractivas.

Palabras clave: aprendizaje; evaluación; química; recurso educativo abierto; usabilidad.

Abstract: The incorporation of ICT in teaching allows students to develop new competencies which are necessary to manage in the society of knowledge. To choose and use web resources, it is important that users can be critic about the material they choose for their learning. The main purpose of this work was the incorporation in the classroom of Open Educational Resources of Chemistry and the evaluation of its usability in charge of the students, using a survey proposed by Spanish authors (Marzal, et al., 2008). The sample was made up of two groups of 15-year-old secondary school students from San Juan, Argentina. The results obtained about the evaluation of usability showed that for the learners, there sources were easy to use and to learn how they worked, the contents were clearly organized and the pictures were appropiate and attractive.

Key words: knowledge; evaluation; chemistry; Open Educational Resources; usability 


\section{INTRODUCCIÓN}

El escenario en que se desarrolla la sociedad actual es sin duda la Sociedad de la Información (SI), donde la educación obtiene una nueva dimensión: "se convierte en el motor fundamental del desarrollo económico y social" (Coll, 2012, p. 114). En la SI las tecnologías de la información y la comunicación (TIC) poseen un papel preponderante ya que sirven como mediador para llegar a la sociedad del conocimiento optimizando la enseñanza y promoviendo mejores aprendizajes.

La capacidad de acceso a la información para adquirir conocimiento que ha sido generado por otros, entre los que se pueden mencionar universidades, instituciones educativas, organizaciones, gobiernos y construir nuevo conocimiento a partir de lo que ya es conocido, se ha potenciado a través de las TIC ofreciendo medios de entrega de la información de forma casi inmediata.

El desafío que actualmente tiene la escuela es ofrecer herramientas cognitivas y el desarrollo de competencias para actuar de modo crítico, creativo, reflexivo y responsable frente a la información y sus usos para la construcción de conocimientos socialmente válidos (Pandiella, 2013).

El acceso y utilización de las TIC requieren continuos y profundos cambios en todos los ámbitos de la sociedad y obviamente en la escuela; ellos exigen una nueva formación de base para los jóvenes y una formación continua, a lo largo de la vida, para todos los ciudadanos. Es necesario que estos cambios estén acompañados, desde la escuela, con actividades que permitan a los alumnos desarrollar nuevas competencias, incorporando la alfabetización digital básica.

Las TIC exigen la adquisición de competencias lectoescritoras y de entendimiento no sólo sobre el contenido sino también sobre el formato y la herramienta que soportan el recurso digital educativo a utilizar.

En este sentido se enfocó la investigación hacia la evaluación por parte de los estudiantes de recursos digitales utilizados en el abordaje de temas de Química, empleando los indicadores de usabilidad propuestos por (Marzal, et al., 2008).

\section{ANTECEDENTES}

El referente teórico de este trabajo aborda los recursos educativos abiertos y la usabilidad de los mismos.

\section{A. Recursos Educativos Abiertos}

La época actual exige un eficaz manejo de las tecnologías de la información y la comunicación. Con el apogeo de las mismas, en las aulas están siendo muy utilizados los recursos educativos abiertos (REA).

Según la Organización de las Naciones Unidas para la Educación, la Ciencia y la Cultura (UNESCO, 2005) en el Forum on the Impact of Open Courseware for Higher Education in Developing Countries, financiado por la William and Flora Hewlett Foundation, los REA constituyen una fuente de acceso libre de materiales educativos digitalizados que pueden ser consultados, utilizados y adaptados por una comunidad de usuarios.

Poseen un esquema de licenciamiento que protege la propiedad intelectual son de uso público, libre y gratuito y disponibles por medios electrónicos a través de internet (Nappa y Pandiella, 2012). Los REA son “... recursos para la enseñanza, el aprendizaje y la investigación que son de dominio público o han sido liberados bajo licencias de propiedad intelectual que permiten su libre uso o reelaboración por otros" (UNESCO, 2005). Los tres elementos que fundamentan y caracterizan a los REA son: educación, tecnología y propiedad intelectual o derecho" (Bonilla Jiménez et al., 2012). 
Según la OCDE (2008) “... el propósito de utilizar los REA en la educación es evidentemente mejorar el aprendizaje, en particular un tipo de aprendizaje que habilita el desarrollo de capacidades individuales y sociales a fin de comprender y actuar".

Para facilitar la diseminación digital de REA existen diversos portales o nodos de distribución digital (Nappa y Pandiella, 2012) de los cuales podemos citar el portal perteneciente a la Universidad de Colorado, PhET.

Este portal ofrece simulaciones entretenidas e interactivas de forma gratuita que permiten a los estudiantes hacer conexiones entre los fenómenos de la vida real y la ciencia subyacente, profundizando sus conocimientos y apreciaciones del mundo.

En este trabajo se utilizaron dos recursos pertenecientes al portal PhET denominados "Sales y solubilidad" y "Construir un átomo". Ellos se encuentran en las siguientes direcciones https://phet.colorado.edu/es_PE/simulation/soluble - $s$ a I t s

$y$
https://phet.colorado.edu/es/simulation/build-anatom respectivamente.

Las fichas catalográficas de los recursos se muestran en las figuras 1 y 2 .

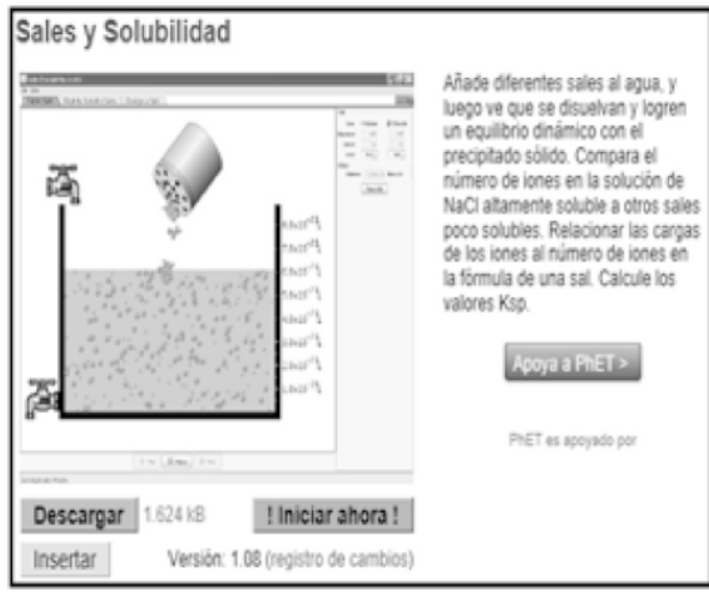

Figura 1. Pantalla de presentación de "Sales y Solubilidad".

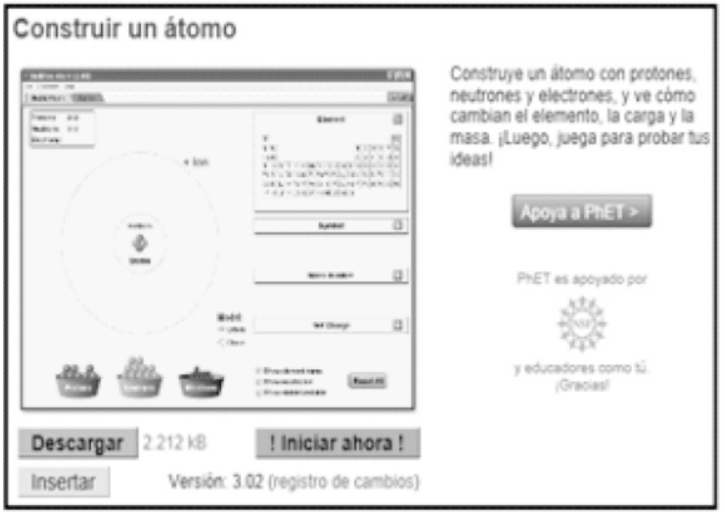

Figura 2. Pantalla de presentación de "Construir un átomo".

\section{B- Usabilidad de recursos educativos abiertos}

La evaluación de los recursos educativos puede realizarse desde diversas perspectivas; así Marzal y Calzada-Prado (2003), elaboraron criterios de análisis para evaluar indicadores de usabilidad, accesibilidad y alfabetización informacional. Estos autores entienden la usabilidad como una serie de factores tales como la amigabilidad del recurso (facilidad en la lectura), flexibilidad, diseño multimedia, la virtualidad e interactividad (propuesta de actividades, autoevaluaciones, foros, archivo de estrategia de los usuarios).

Los recursos seleccionados con fines educativos deberán cumplir una serie de requisitos de usabilidad y que posibiliten una óptima experiencia de aprendizaje (Pandiella, 2013).

El concepto de usabilidad presentado por la norma ISO/IEC 9241 Ergonomic requirements for visual display terminals (ISO/IEC 9241, 1998), en su undécima parte, Guidance for usability, en la que se define a la usabilidad como el grado con que un determinado producto, en un contexto de uso específico, permite al usuario alcanzar sus objetivos con eficacia, eficiencia y satisfacción.

En los ambientes virtuales, la usabilidad resalta como cualidad de un sistema, aplicación, herramienta u 
objeto que indica la facilidad con que se puede utilizar. Implica la posibilidad de usar, que sus opciones (si las tiene) sean fáciles de recordar, que sea entendible, que su manipulación sea muy intuitiva, etc. (Nielsen, 2000).

Marzal et al. (2008) proponen para la evaluación de la usabilidad de un REA, tres categorías: Captación, vinculada al mecanismo cognitivo de la atención; Fidelización, vinculada a la percepción y Capacidad alfabetizadora, vinculada con la memoria.

\section{METODOLOGÍA}

Se trabajó con dos muestras de sendas instituciones escolares de gestión estatal. Una de ellas, constituida por 21 alumnos, trabajó con el recurso "Sales y solubilidad" (Figura 1) y la otra, compuesta por 28 alumnos, hizo uso del recurso "Construir un átomo" (Figura 2). Los dos grupos pertenecen al 3으o año de Nivel Secundario de escuelas de la Provincia de San Juan, República Argentina y la edad promedio de ellos al momento de la aplicación del cuestionario era de 15 años. Los estudiantes interactuaron con los REA seleccionados y luego contestaron el cuestionario propuesto por Marzal et al. (2008) compuesto por 41 preguntas referenciadas con las siglas P1, P2,..., P41.

\section{RESULTADOS}

Los resultados del cuestionario para evaluar la usabilidad de los recursos se muestran en las Tablas 1 y 2 donde las casillas con sombreado negro representan $\mathrm{SI}$, en blanco representan $\mathrm{NO}$ y en gris representa que no contesta o manifiesta dudas NS/NC.
Tabla 1. Resumen de resultados para evaluar la usabilidad del REA "Sales y Solubilidad".

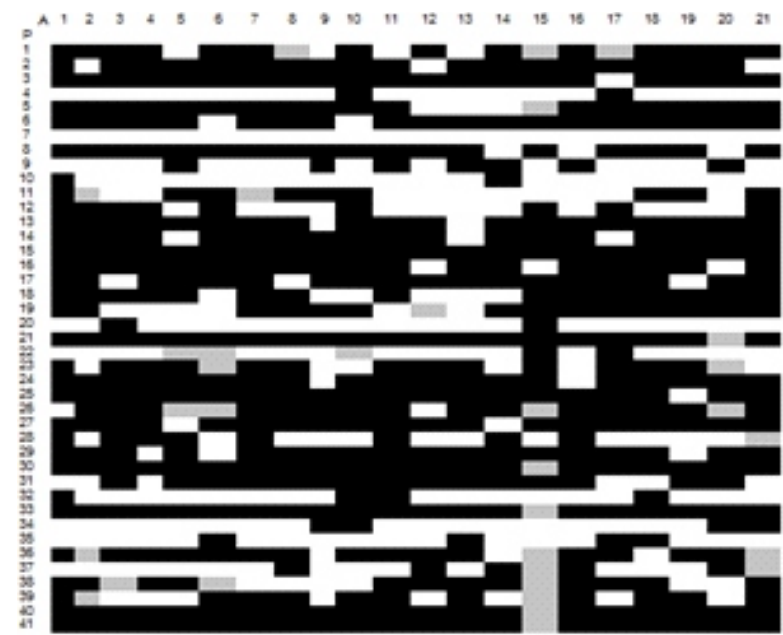

Tabla 2. Resumen de resultados para evaluar la usabilidad del REA Construir átomos.

Los resultados obtenidos, discriminados según las categorías propuestas por Marzal et al. (2008) y por recurso educativo, se muestran en los Gráficos 1, 2, 3, 4,5 y 6 .

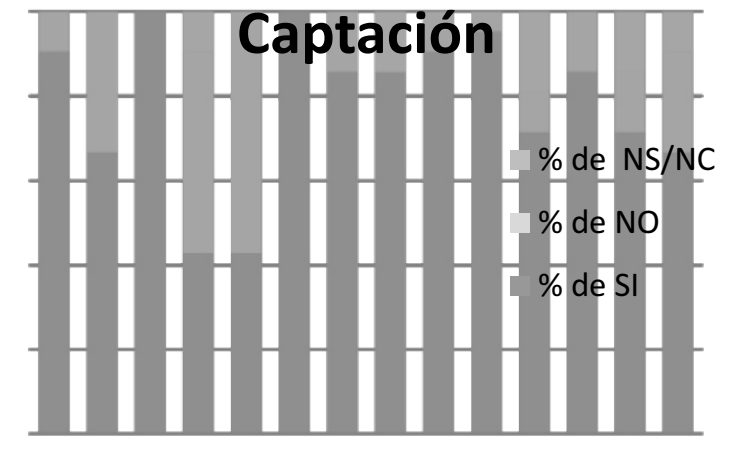

Gráfico 1. Captación para el REA "Sales y solubilidad”. 


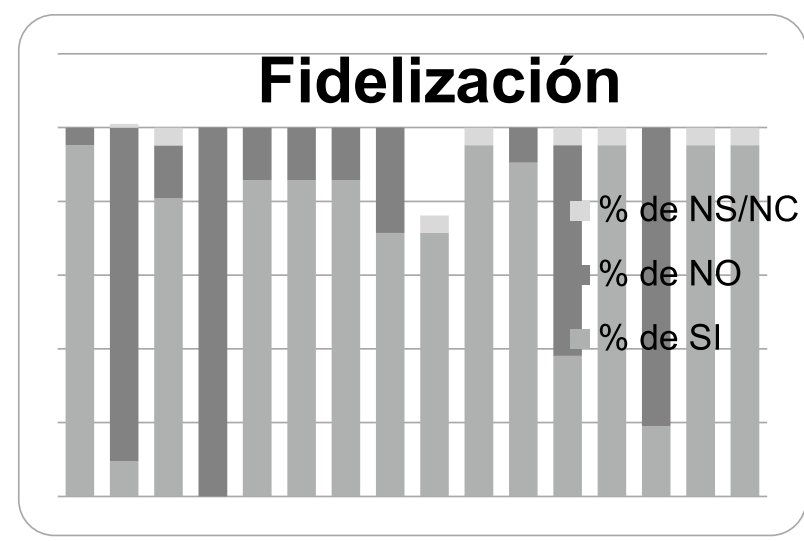

Gráfico 2. Fidelización para el REA "Sales y solubilidad".

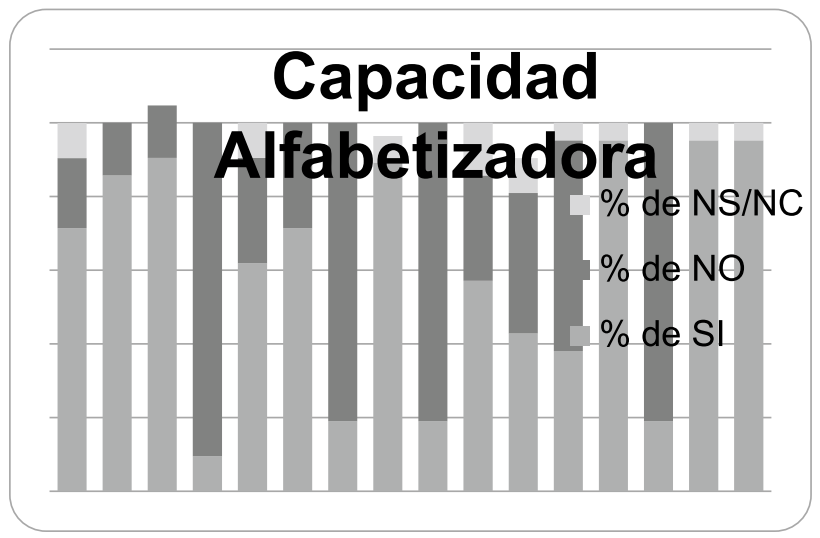

Gráfico 3. Capacidad alfabetizadora para el REA "Sales y solubilidad".

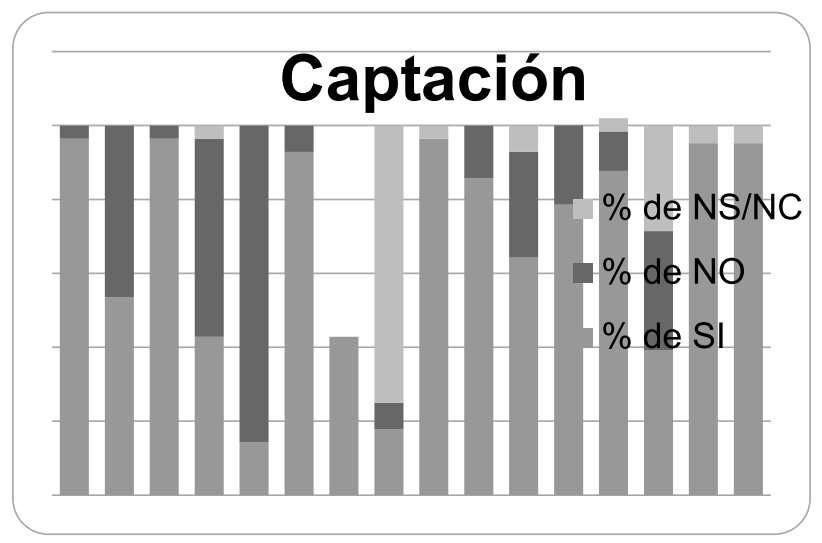

Gráfico 4. Captación para el REA "Construir átomos".

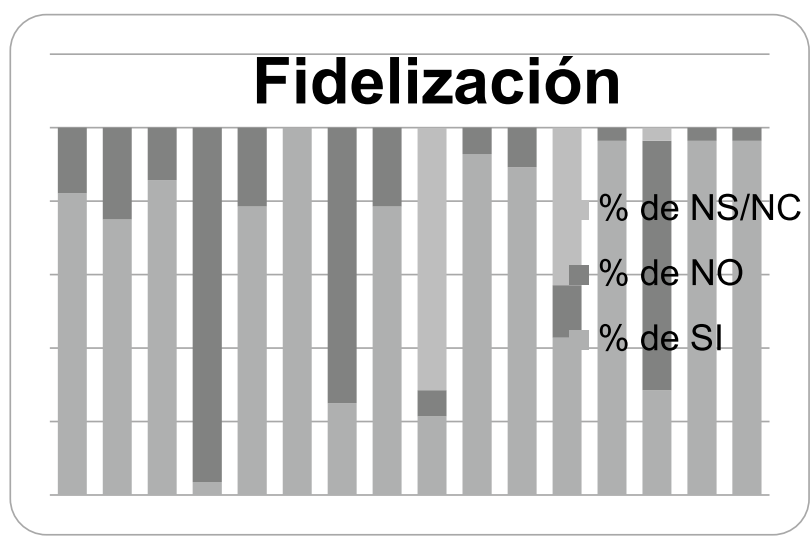

Gráfico 5. Fidelización para el REA "Construir átomos".

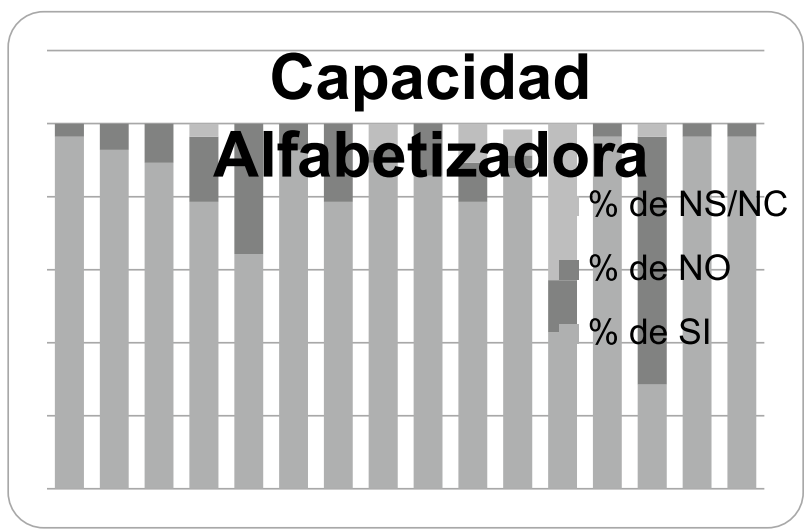

Gráfico 6. Capacidad alfabetizadora para el REA "Construir átomos".

Para ambos recursos los datos referidos a Captación demuestran que, en general, todas las preguntas tienen alto grado de acuerdos, resultando atractivo su aspecto general (P6), aunque expresan la dificultad para comprender el lenguaje usado como también del contenido del recurso (P9 y P37). El manejo de los recursos ha resultado fácil y no requiere mucho esfuerzo para la mayoría de los alumnos (P36, 71\% en el caso de "Sales y Solubilidad" y $87 \%$ en el caso de "Construir un Átomo", el diseño les ha parecido lo suficientemente claro para utilizar y localizar la información en él (P15, 100\% y 93\%). Además los recursos responden adecuadamente a su ritmo de navegación (P25) aunque el recurso "Construir un 
átomo" no posibilita la navegación entre contenidos cada vez más específicos (P22, 18\%).

En cuanto a la categoría Fidelización se observa que ambos recursos se perciben efectivos para aprender ya que disponen de un Mapa del sitio (P3), son pertinentes las cabeceras en las que aparecen los contenidos que se pueden consultar (P5) y no contienen publicidad que obstaculice el acceso a los contenidos (P7) y también en ambos recursos la organización de los contenidos les resulta clara (P21) y las imágenes utilizadas son apropiadas e ilustrativas, obteniéndose porcentajes similares y mayores del $90 \%$. También la mayoría de los estudiantes volverían a visitar el recurso y lo recomendarían a otros compañeros (P40 y P41).

Con respecto a la Capacidad alfabetizadora, los estudiantes evalúan los recursos mayoritariamente en forma positiva en lo referente a la identificación de la autoría y calidad de los contenidos (P2 y P39), los recursos presentan información de distinto tipo como: texto, imágenes, animaciones, íconos (P31), y se percibe eficaz (P33) por disponer de actividades que se plantean como juego o entretenimiento. En el caso del recurso "Sales y Solubilidad" los estudiantes estiman que el recurso no permite visualizar los enlaces que se van siguiendo de manera que puedan ver la secuencia de los movimientos (P20). Para el mismo recurso los estudiantes indican que no plantea actividades de diferente complejidad en función de los resultados obtenidos (P32) y está de acuerdo sólo un $43 \%$ en que el recurso les brinda seguridad en relación a la calidad de sus contenidos (P39).

\section{CONCLUSIONES}

Los estudiantes han desarrollado ciertas capacidades de acceso a la información para adquirir conocimiento como así también criterios para evaluar los recursos educativos propuestos.
La evaluación que realizaron los estudiantes a los dos recursos del portal PhET, dio como resultado que ambos se mostraron aptos y eficaces en la mayoría de los aspectos relacionados con el mecanismo cognitivo de la atención (Captación), la percepción (Fidelización) y la memoria (Capacidad alfabetizadora).

AGRADECIMIENTOS Las autoras agradecen a la Universidad Nacional de San Juan, Argentina, que financió la investigación. 


\section{REFERENCIAS}

Bonilla Jiménez, M.; García Gutiérrez F. J. y González Gómez, L. J. (2012). Incorporación de Recursos Educativos Abiertos Como medio para promover el aprendizaje significativo a nivel universitario: UN estudio de casos. En S. Ramírez Montoya y J. V. Burgos Aguilar. Coord. Recursos Educativos Abiertos en Ambientes Enriquecidos con Tecnología Innovación en la Práctica Educativa.

Coll, C. (2012). Aprender y enseñar con las TIC: expectativas, realidad y potencialidades. En $\mathrm{R}$. Carneiro, J. C. Toscano, T. Díaz, Coord. Los desafíos de las TIC para el cambio educativo. Madrid. España: Editorial Santillana.

Construir UN átomo. Recuperado de https://phet.colorado.edu/es/simulation/build-anatom/2012/07/18.

International Standards Organization Ergonomic requirements for visual display terminals ISO/IEC 9241. (1998).

Marzal, M. A.; Calzada-Prado, F. J. (2003). UN análisis de necesidades y hábitos informativos de estudiantes universitarios en Internet. Binaria, 3. Recuperado de http://www.uem.es/binaria/in.html.

Marzal, M.; Calzada-Prado, J. y Vianello, M. (2008).Criterios para la evaluación de la usabilidad de los recursos educativos virtuales: UN análisis desde la alfabetización en información. Information research, 13 (4). Recuperado de http://informationr.net/ir/134/paper387.html/2012/04/25

Nappa; N. R y. Pandiella, S. B (2013). Estudio y aplicación de objetos de aprendizaje a través Del USO de recursos educativos abiertos. EDUTEC, Revista Electrónica de Tecnología Educativa. Núm. 39, pp. 117. Recuperado de

http://edutec.rediris.es/Revelec2/Revelec39/estudio _aplicacion_obejtos_aprendizaje_recursos _educativos_abiertos.html./2013/03/21.

Nielsen, J. (2000).Usabilidad: Diseño de sitios Web. Madrid. España: Prentice Hall.

OCDE. (2008). El conocimiento libre y los Recursos Educativos Abiertos. Recuperado de http://www.oecd.org/dataoecd/44/10/42281358.pd f./2012/09/30.

Pandiella, S. (2013). Las decisiones Del docente y los aprendizajes en sus alumnos. Seminario en Formación de educadores en ambientes a distancia para el desarrollo de competencias en el USO de REAs.

Sales y solubilidad. Recuperado de https://phet.colorado.edu/es_PE/simulation/soluble -salts/2012/07/18.

UNESCO (2012). Hacia las sociedades Del c o n o c im i e n t o. R e c u p e ra do d e http://unesdoc.unesco.org/images/0014/001419/1 41908s.pdf. 2005./01/11/2012 